\title{
The Effect of Sequence Repeat Time on Auditory Cortex Stimulation During Phonetic Discrimination
}

\author{
N. J . Shah, S. Steinhoff, S. Mirzazade, O. Zafiris, M.-L. Grosse-Ruyken, L. J äncke,* and K. Zilles \\ Institut für Medizin, Forschungszentrum J ülich GmbH, D-52425 J ülich, Germany; and *Institute of General Psychology, \\ Otto-von-Guericke University Magdeburg, Magdeburg, Germany
}

Received December 20, 1999

Acoustic noise generated by the MR scanner gradient system during fMRI studies of auditory function is a very significant potential confound. Despite these deleterious effects, fMRI of the auditory cortex has been successful and numerous practitioners have circumvented the problem of acoustic masking noise. In the context of auditory cortex fMRI, the sequence repeat time (TR) has the effect of altering the length of time during which the scanner is quiet. Indeed, the move to whole-brain fMRI makes the problem of acoustic noise more acute and points to the need to examine the role of TR and its influence on the BOLD signal. The aim of this study was to examine the effect of varying the TR time on activation of auditory cortex during presentation and performance of a phonetic discrimination task. The results presented here demonstrate that the influence of sequence repeat time is considerable. For a short repeat time it is likely that the noise from the scanner is a significant mask and hinders accurate task performance. At the other extreme, a repeat time of $9 \mathrm{~s}$ is also suboptimal, probably due to attentional effects and lack of concentration and not least because of the longer overall measurement times. The results of this study point to a complicated interplay between psychophysical factors as well as physical parameters; attention, acoustic noise, total duration of the experiment, consideration of the volume of acquisition, and overall difficulty of the task have to be assessed and balanced. For the paradigm used here, the results suggest an optimal TR of around $6 \mathrm{~s}$ for a 16-slice acquisition. 2000 Academic Press

\section{INTRODUCTION}

The application of fMRI to study activation of human auditory cortex, despite the presence of acoustic noise generated by the gradient system of the MR scanner (Bandettini et al., 1998; Edmister et al., 1999; Hall et al., 1999; Shah et al., 1999; Talavage et al., 1999), has become widespread (Belin et al., 1999; J äncke et al., 1999; Wessinger et al., 1997; Millen et al., 1995; Binder et al., 1994). Regional changes of the nuclear magnetic resonance signal during brain activity are brought about by changes in regional blood flow and are taken to reflect underlying neural activity. It is generally accepted that increased blood flow and oxygenation at the capillary venous level in activated tissue lead to decreased intravoxel dephasing, resulting in increased signal. The feasibility of fMRI as a tool for the study of auditory perception processes has been shown by a number of research groups (e.g., Belin et al., 1999; Binder et al., 1994, 1995, 1996a, 1996b, 1997; Edmister et al., 1999; Hall et al., 1998; J äncke et al., 1998; Millen et al., 1995; Woodruff et al., 1996). However, many early studies did not concentrate on optimization of the various paradigms that were employed. Mainly, it was only later that the focus of attention shifted to the elucidation of the more elementary stimulus variables such as intensity (Binder et al., 1994; J äncke et al., 1998), duration (J äncke et al., 1999), and the influence of acoustic noise (Bandettini et al., 1998; Edmister et al., 1999; Hall et al., 1999; Shah et al., 1999; Talavage et al., 1999; Cho et al., 1997, 1998). The increase in sophistication of the basic $\mathrm{fMRI}$ experiment demanded by studies designed to probe language and higher cognitive function has required a reexamination of these more elementary variables. J äncke et al. (1998) have shown that the intensity of the stimulus is an important parameter and has to be carefully controlled. The rate of presentation of auditory stimuli has also been shown to affect the elicited BOLD response while stimulus duration did not affect the BOLD response significantly (Binder et al., 1994; J äncke et al., 1999). Several recent studies have also attempted to deal with the question of just how much acoustic noise affects the response of the auditory cortex, either qualitatively (Bandettini et al., 1998; Hall et al., 1999; Shah et al., 1999; Cho et al., 1997, 1998), or quantitatively (Talavage et al., 1999). Some of those latter studies have instigated the development of methods to splice the presentation of the auditory stimuli with the acoustic noise from the scanner, with the resultant methods generically termed as "clustered volume acquisition" 
(Van de Moortele et al., 1998; Edmister et al., 1999) and "sparse temporal sampling" (Hall et al., 1999). Both studies have raised the issue of the sequence repeat time, and this work, which has been presented in parallel to the aforementioned studies, in the form of a conference abstract (Shah et al., 1998), attempts to deal with the self-same issue but for the specific case of phonetic discrimination. Edmister et al. note that clustered volume acquisition method is typical on Siemens scanners; such a scanner with clustered volume acquisition was used for this study.

Although the influence of TR on the MR signal is well-known, in the context of fMRI, the choice of TR is often a compromise between the length of the entire experiment and a desire for higher signal-to-noise ratios in the acquired images. The acquisition of a time series of EPI images may be viewed as a basic excitation-recovery problem where the magnetisation in the direction of the main magnetic field directly before a $90^{\circ}$ pulse (excluding the first pulse) is proportional to $\mathrm{M}_{0}\left[1-\exp \left(-\mathrm{TR} / \mathrm{T}_{1}\right)\right]$, where $\mathrm{M}_{0}$ is the initial magnetization. If $T R \ll T_{1}$, then $T_{1}$ relaxation effects lead to a falling overall signal intensity in the first few images of the time series until a steady state is established. Further, to maximize the steady-state signal when using a $T R \ll T_{1}$, the optimal flip angle is not $90^{\circ}$. Generally, the optimal flip angle (the Ernst angle) should be employed and is given by the well-known relation $\theta=\cos ^{-1}\left[\exp \left(-\mathrm{TR} / \mathrm{T}_{1}\right)\right]$. The influence of TR on the BOLD response is a more complicated issue and involves psychophysical factors such as attention as well as those briefly discussed above. The purpose of this study is to try and answer this question. The use of auditory paradigms brings with it the need to consider additional factors since the noise of the scanner itself elicits auditory activation, a topic which has been discussed el oquently by Bandettini et al . (1998), Hall et al . (1999), and Edmister et al. (1999). Indeed, the move to whole-brain fMRI makes the problem of acoustic noise all the more acute and points to the need to examine the role of TR and its influence on the resulting BOLD signal. Varying the TR is expected to lead to differences in the intervolume noise interference. The acquisition of more slices to achieve whole-brain coverage will lead to differences in the intravolume noise interference, if the number of acquired slices is large and the clustered volume acquisition time exceeds the BOLD response onset time. Although we have not used whole-brain coverage here, we note that there is an increasing tendency amongst researchers to do so, and we there fore comment briefly on the consequences of so doing. We examine here how varying the repeat time of the MR sequence (clustered acquisition using EPI) influences activation of auditory cortex during presentation and performance of a phonetic discrimination task. Throughout the study the number of acquired slices
(16), the total number of scans (48), and the slice acquisition time have been kept constant.

\section{MATERIALS AND METHODS}

Five right-handed, healthy subjects (all male), with no history of neurological or audiological illness, were studied while performing a phonetic discrimination task. The entire brain was scanned in 3-D to obtain anatomical images using a high-resolution, $\mathrm{T}_{1-}$ weighted MP-RAGE pulse sequence (magnetizationprepared, rapid acquisition gradient echo) with the following parameters: TR (repetition time) $=11.4 \mathrm{~ms}$; TE (echo time) $=4.4 \mathrm{~ms} ; \theta$ (flip angle) $=15^{\circ} ; 1$ excitation; field-of-view (FOV) $=230 \mathrm{~mm}$; matrix $=200 \times$ 256; 128 sagittal slices with $1.25-\mathrm{mm}$ slice thickness. Echo Planar I maging (EPI) was performed on a Magnetom Vision 1.5 Tesla scanner (Siemens Medical Systems $\mathrm{GmbH}$, E rlangen, Germany) equipped with a gradient booster system; the standard radiofrequency head coil was used for transmission of rf and signal reception. Pulse sequence parameters were as follows: gradient echo EPI; TE $=66 \mathrm{~ms} ; \mathrm{FOV}=200 \times 200 \mathrm{~mm}$; $\theta=90^{\circ}$; matrix size $=64 \times 64$; pixel size $=3.125 \times$ $3.125 \mathrm{~mm}$; slice thickness $=3.0 \mathrm{~mm}$; interslice gap $=$ $0.3 \mathrm{~mm} ; 16$ slices. The fMRI paradigm consisted of a preceding baseline of 9TR followed by three repetitions of an activation-rest cycle and a final activation period, with each activation and rest component being $6 T R$ in length. Each volunteer performed six experiments during the whole measurement procedure. The TR of successive experiments on each volunteer was varied, in a pseudo-random manner, and was either 3, 5, 6, 7, 8, or 9 s. Using a left hemispheric, sagittal slice from the anatomical MR images, 16 oblique slices were oriented along the course of the AC-PC line. Subjects gave informed, written consent following a full explanation of the nature and risks of the research, according to a protocol approved by the Ethics Committee of the Heinrich-Heine University of Düsseldorf.

During scanning the room lights were dimmed and the subjects kept their eyes open. Auditory stimuli were presented binaurally using a digital playback system, a magnetically shielded transducer system and air conduction through paired plastic tubes. The air conduction system terminated in tightly occlusive headphones allowing unimpeded conduction of the stimulus with good suppression of ambient scanner noise by about $15 \mathrm{~dB}$. The sound pressure level (SPL) of the stimulus was on average $90 \mathrm{~dB}$ SPL and remained constant across experiments and subjects. This stimulus intensity level was chosen on the basis of previous experiments which demonstrated excellent auditory cortex activation with the same paradigm (J äncke et al., 1998). The intensity of the stimuli was determined outside the scanner using an artificial head (Bruel \& Kjaer KA637) by measuring the mean signal intensity 
during a 30-s epoch. The average intensity of the scanner noise was approximately $75 \mathrm{~dB}$ SPL after attenuation by the headphones.

\section{Auditory Stimuli and Experimental Conditions}

Stimuli were 16-bit, digitally sampled, consonantvowel (CV) syllables (/ka/, /ta/, /pa/, /ga/, /da/, /ba/) recorded by a trained phonetician. The onset, duration, intensity, and fundamental frequency of the stimuli were edited and synchronised by means of a speech editor. The criterion for temporal alignment of the syllables was the onset of articulatory release. Each syllable started with zero intensity of 43-ms duration before start of the articulation and stopped with zero intensity of 475- to 552-ms duration after vowel end. This manipulation served to remove clicks at the beginning and end of digital-to-analogue conversion. The syllables were digitally produced and loaded into the working memory of the PC used for stimulation. Depending on voice onset times, the duration of syllables ranged from 405 to $482 \mathrm{~ms}$. The duration of the entire presentation (syllable, leading, and trailing intervals of zero intensity) was thus exactly 1000 ms. These syllables were randomized and arranged in syllable pairs (each pair containing the same syllables for binaural presentation). Each of the six CV syllables occurred with equal frequency. Since all possible syllables were equiprobable, one-sixth of the trials contained the syllable/ta/, which served as target syllable in the experiments. The interval between syllable pairs was $1 \mathrm{~s}$. During the $\mathrm{TR}=3,5,6,7,8,9 \mathrm{~s}$ experiments, the total number of syllable pairs pre sented was $72,120,144,168,192$, and 216 , respectively. The order of the experimental conditions was randomized across the six subjects.

Subjects were instructed to respond to the occurrence of each target syllable by briefly pressing a button with the right index finger. These responses were recorded by the stimulus presentation software and allowed subsequent analysis of performance rate and reaction time. The stimulation program was written using MEL Professional (Psychology Software Tools, Inc. Pittsburgh, PA) and the button-press functioned by interrupting a light beam, which was carried out of the shielded magnet room for detection using a lightcarrying fiber.

\section{Data Analysis and Statistical Parameter Mapping}

I mage analysis was performed on a SPARC UItra 1 workstation (Sun Microsystems, Palo Alto, CA) using MATLAB (Version 4.2c, The Mathworks, Inc., Natick, MA) and SPM 96 software (Statistical Parametric Mapping, The Wellcome Department of Cognitive Neurology, London; http://www.fil.ion.ucl.ac.uk/spm). The first three images of each time-series, during which the MR signal reaches a steady-state, were discarded.
These three images were discarded from all analyses not only to allow the MR signal to reach equilibrium, but also because subjects often jerk their heads in response to the sudden noise of the scanner; the first image if included in the data analysis has the potential to be a serious confound. The 48 remaining volume data sets of each condition were automatically realigned to the first image to correct for head movement between scans. All data sets were motion-corrected using the coregister and reslice algorithms in SPM 96 (Friston et al., 1996, 1995a, 1995b, 1994a, 1994b; Poline et al., 1995; Worsley et al., 1995).

Given the relatively low spatial resolution of the EPI images, bilinear interpolation was employed during the motion correction procedure. Significantly activated voxels were found by using SPM96 software based on the "General Linear Model" and the theory of random Gaussian fields for the analysis of time-series data (Friston et al., 1995a, 1995b). The resulting SPM (t) map was used for further statistical analysis. Image sets for the six conditions were then coregistered to the 3-D anatomical data using MPItool (Pietrzyk et al., 1990a, 1990b), the AC point identified, and transformed into Talairach space, using the AC point, thereby allowing direct cross-reference to atlas topography. Transformed functional data sets from each subject were smoothed with a Gaussian filter (rootmean-square radius $=6 \mathrm{~mm}$ ) to compensate for normal variation in sulcal/gyral anatomy across subjects. The time series were high-pass filtered to remove low frequency artifacts due to cardio-respiratory and other cyclical components.

Statistical analysis was performed for single subjects and the stereotaxically normalized fMRI time-series data from the six subjects were also pooled for further analysis. The alternating periods of "baseline" and "activation" were modelled using a smoothed, delayed boxcar reference vector.

Because the activated clusters covered a wide range of auditory cortex, we defined vol umes of interest (VOI) in each hemisphere to account for the diversity of the auditory cortex (primary, immediate, and secondary auditory cortex). These VOI s were defined according to the Talairach atlas, prominent sulcal, and gyral landmarks, and previous articles on human PET and fMRI studies concerning auditory cortex activation (Zatorre et al., 1992; Binder et al., 1997; J äncke et al., 1998). The anterior auditory cortex (A1) was defined as $\pm 28<$ $x< \pm 76,-36<y<-8,8<z<16$, the middle auditory cortex (A2) was defined as $\pm 76<x< \pm 28$, $-40<y-36,4<z<20$, and the posterior auditory cortex (A3) was defined as $\pm 76<x< \pm 32,-48<y$ $-40,0<z<20$ (for left and right auditory cortex negative or positive $x$ coordinates were used). The volumes of these VOI s were $10.7 \mathrm{ml}(\mathrm{A} 1), 3.1 \mathrm{ml}(\mathrm{A} 2)$, and 7.7 (A3). For each VOI, the number of significantly activated voxels and the mean t score representing the 
L-H1

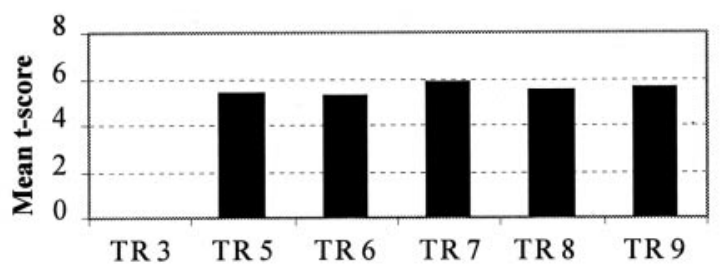

L-PTa

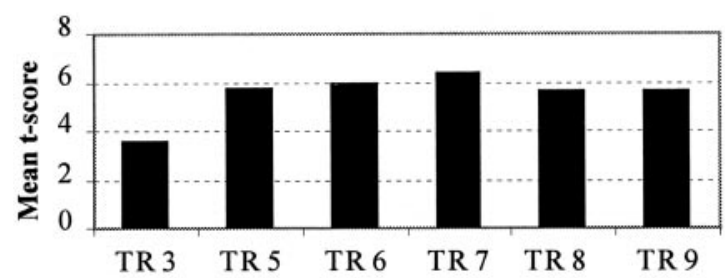

L-PTp

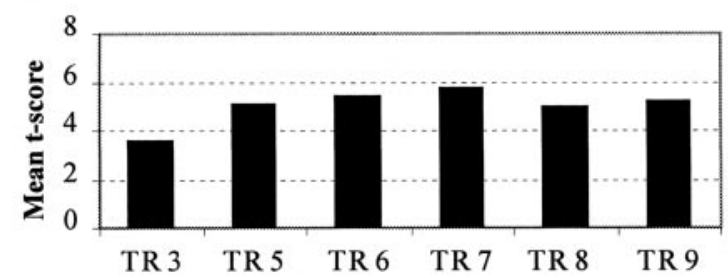

R-H1

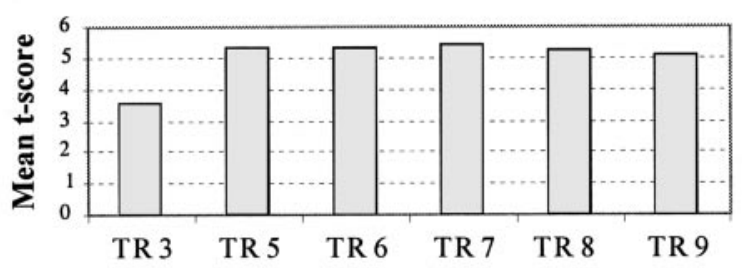

R-PTa

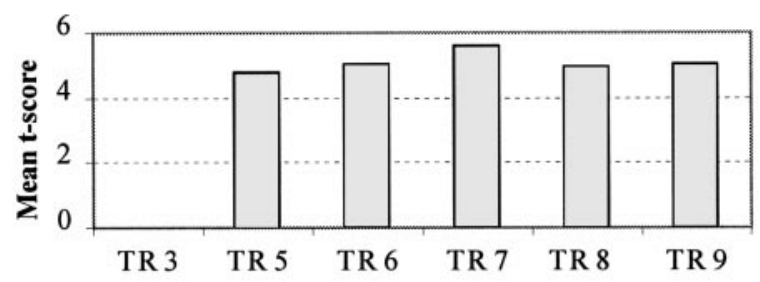

R-PTp

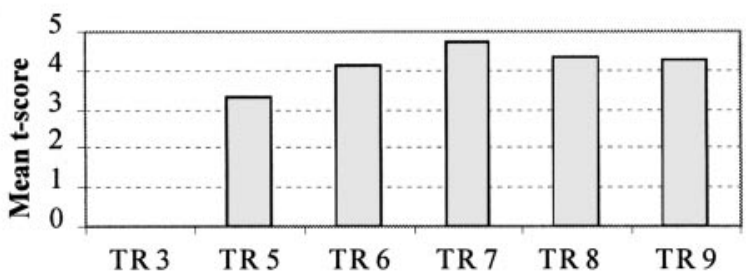

FIG. 1. The mean $t$ scores from the group analysis obtained for each of three VOIs, Heschl's gyrus, the anterior and posterior portions of the Planum Temporale. Results for each hemisphere are shown separately for the six experimental conditions. For both hemispheres, there is an initial increase of the mean t score from TR $=3 \mathrm{~s}$ to $T R=7 \mathrm{~s}$, followed by a gradual levelling off to TR $=9 \mathrm{~s}$. See text for a more detailed description of the VOIs.

normalised difference between the ON and OFF conditions were calculated.

\section{RESULTS}

During the auditory phonetic discrimination task we observed differing extents of activation in primary and secondary auditory cortex of all volunteers and across all conditions. This is consistent with the findings of previous fMRI studies. From the group analysis, the maximum t scores of the VOIs activated for each of the six conditions were determined as described above. The t scores for the six conditions are shown in Fig. 1, separately for the left and right hemispheres, for three VOIs (A1, A2, and A3). There is a gradual increase of the $t$ score as the TR increases from 3 to $7 \mathrm{~s}$ and thereafter the t score exhibits a levelling off to TR = 9 s. This trend was confirmed by a series of trend analyses with TR as predictor and mean t scores as dependent variables. These trend analyses revealed significant positive quadratic trends for all VOIs $\left(\mathrm{R}^{2}\right.$ ranging from 0.95 to 0.99 ). Here, $R^{2}$ expresses the goodness-of-fit of the trend curves ranging from 0 to 1 , where 0 indicated no fit and 1 indicates an optimal fit. The mean number of voxels passing a threshold of $t=$ 4 was also calculated (Fig. 2) and subjected to trend analysis. For the mean t score, there is an increase in the number of activated voxels with an increase in TR for all regions ( $\mathrm{R}^{2}$ ranging from 0.79 to 0.98 ). However, it is evident from the figures that the mean number of activated voxels as well as the mean t score is lowest for the TR3 condition while there are only subtle differences between these measures obtained during the other TR conditions. Therefore, we performed additional trend analysis by excluding the measures obtained for TR3. These analyses also revealed substantial quadratic trends with $\mathrm{R}^{2}$-values ranging from 0.39 to 0.92 . A closer inspection of the data obtained 
L-H1

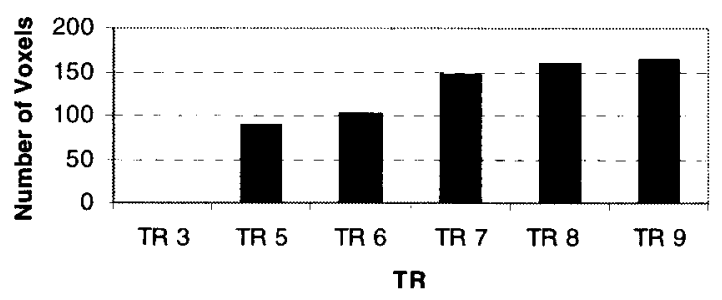

L-PTa

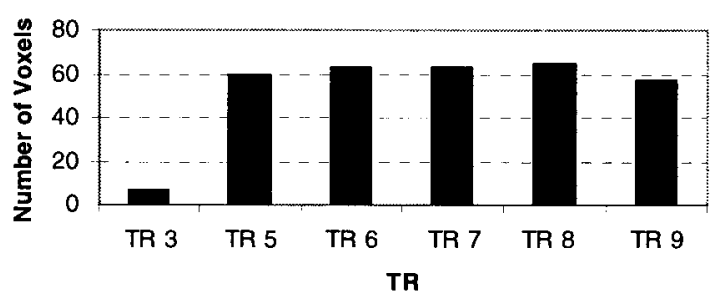

L-PTp

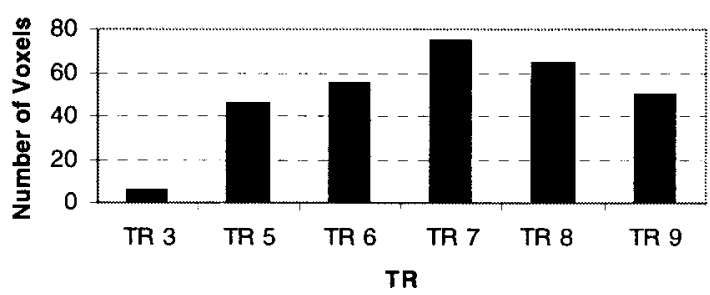

R-H1

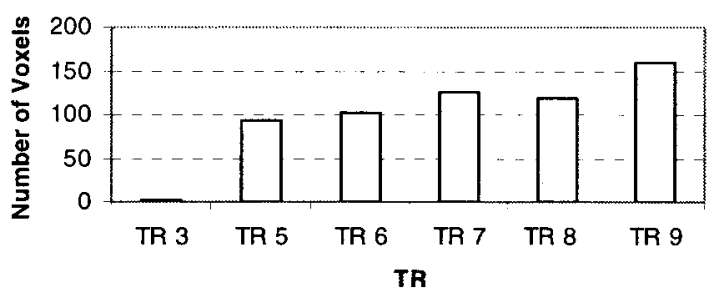

R-PTa

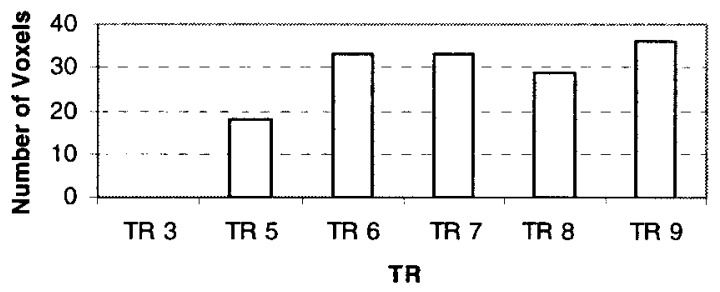

R-PTp

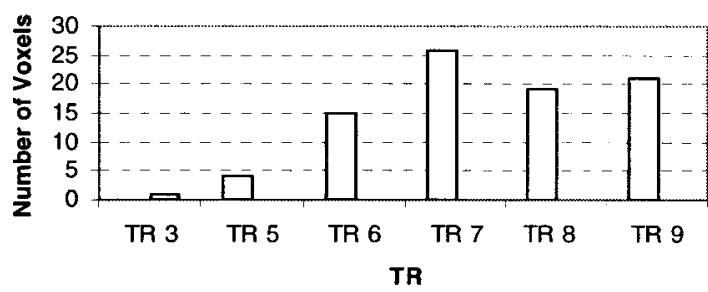

FIG. 2. The mean number of activated voxels passing a threshold of $z=4$, obtained from the group analysis, for each of three VOIs, Heschl's gyrus, the anterior and posterior portions of the Planum Temporale. Results for each hemisphere are shown separately for the six experimental conditions. As for the mean $\mathrm{t}$ scores, for regions $\mathrm{H} 1$ and $\mathrm{Pt}_{\mathrm{a}}$, there is a gradual increase in the size of the activated volume with increasing TR. For region $\mathrm{PT}_{\mathrm{p}}$, there appears to be a maximum at around TR $=7 \mathrm{~s}$, followed by a gradual decline to TR $=9 \mathrm{~s}$; again, this holds for both hemispheres.

shows that the mean t-scores were largest during the TR7 condition. F or the mean number of activated voxels we found on the posterior auditory cortex (A3) peak activation during the TR7 condition.

Figure 3 shows the glass brain projections from the SPM analysis and schematically represents the mean activated areas on stereotaxically normalised brains. The regions of activation encompass the primary and secondary auditory cortices and show an increase in the volume of the activated regions as TR increases. Performance during each experiment was recorded and subsequently analysed for correctness of response and reaction time. The percentages of correct responses (number of correct responses as a fraction of the total number of presentations for each condition, summed across the volunteers) are shown in Fig. 4. It can be seen that there was an improvement in performance as the TR increased from 3 to $6 \mathrm{~s}$. There then follows an abrupt fall in performance for the TR $=7 \mathrm{~s}$ condition and then a further gradual increase to TR $=9 \mathrm{~s}$. The corresponding reaction times are shown in Fig. 5 . There is an apparent small drift downward from 3 to
$7 \mathrm{~s}$ and then a gentle increase to the TR $=9 \mathrm{~s}$ condition. The standard deviation, as indicated by the error bars is, however, large, and significant changes in reaction time, if any, are small. Subjecting these performance scores to trend analysis revealed similar trends as for the haemodynamic responses (correct performance: positive quadratic trend, $\mathrm{R}^{2}=0.44$, reaction time: negative quadratic trend, $R^{2}=0.80$ ). In general, both performance measures resemble the haemodynamic responses in all VOIs as is also indicated by the strong correlations between these performance measures and the haemodynamic responses (correct performance with haemodynamic responses for all VOIs: correlation coefficient, $r$, ranges from 0.65 to 0.66 ; reaction time with hemodynamic responses for all VOIs: $r$ ranges from -0.64 to -0.83 ).

\section{DISCUSSION}

The results of these experiments demonstrate an increase in the t score as TR is increased from 3 to $7 \mathrm{~s}$ followed by a levelling off to TR $=9 \mathrm{~s}$. This trend is 


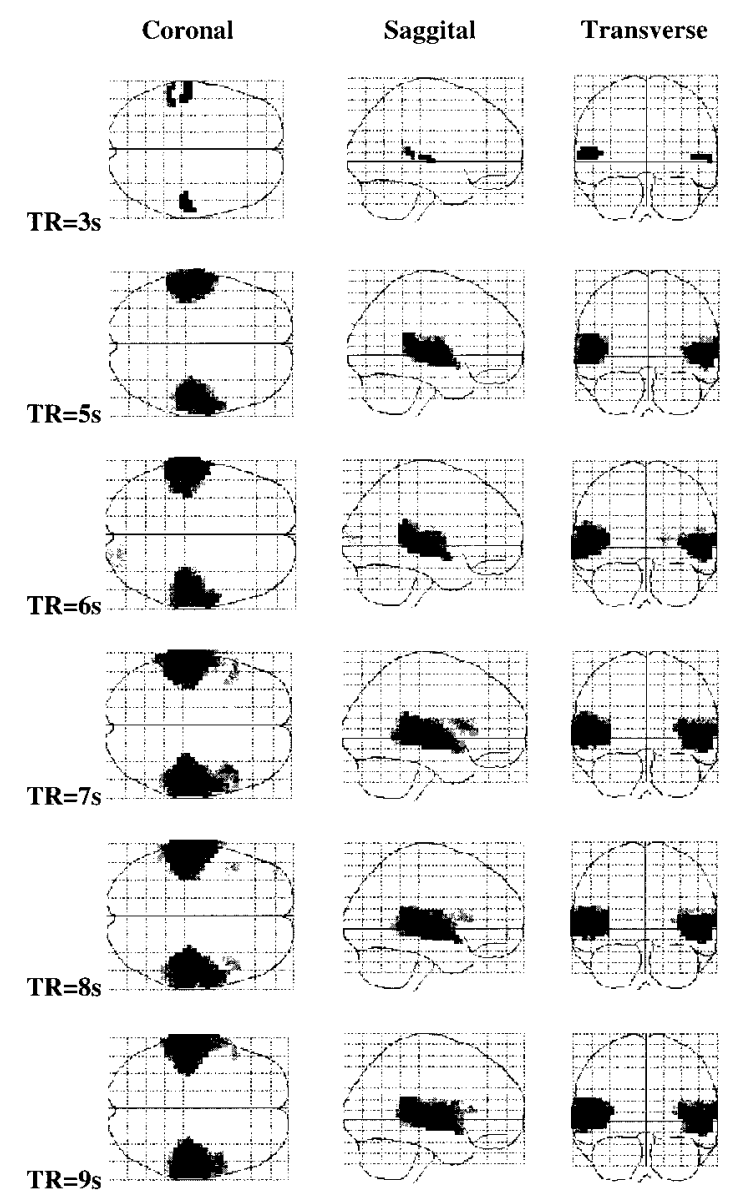

FIG. 3. Schematic representation of the mean activated areas on stereotaxically normalised SPM glass brains. The sagittal, coronal and transverse views are shown for each of the six conditions, namely, TR $=3,5,6,7,8$, and 9 s.

more or less reflected in the change of the volume of the activated regions as a function of TR. The TR $=3 \mathrm{~s}$ condition suffers from the fact that the acoustic noise generated by the gradient system of the MR scanner is present for about 2/3 of the imaging time and it is therefore not surprising that subjects reported difficulty in performing the task. These difficulties are reflected in the poor performance figures for this condition. During the whole experiment, only 72 target stimuli were presented, at a rate of $1 \mathrm{~Hz}$, which was held constant throughout the study. For the TR $=3 \mathrm{~s}$ condition, during the ON phase of the paradigm 18 target stimuli were presented. This is the same number as that used by Binder et al. (1994) with which they achieved a robust BOLD response. There is no evidence in the literature to suggest that this total number of target stimuli is too low to evoke an adequate bold response and therefore the low t scores in the TR $=3 \mathrm{~s}$ condition cannot be attributed to this. Effects due to partial saturation of the MR signal brought about by the short TR may also be ruled out as a possible explanation of the low $t$ scores based on the observation that
Performance

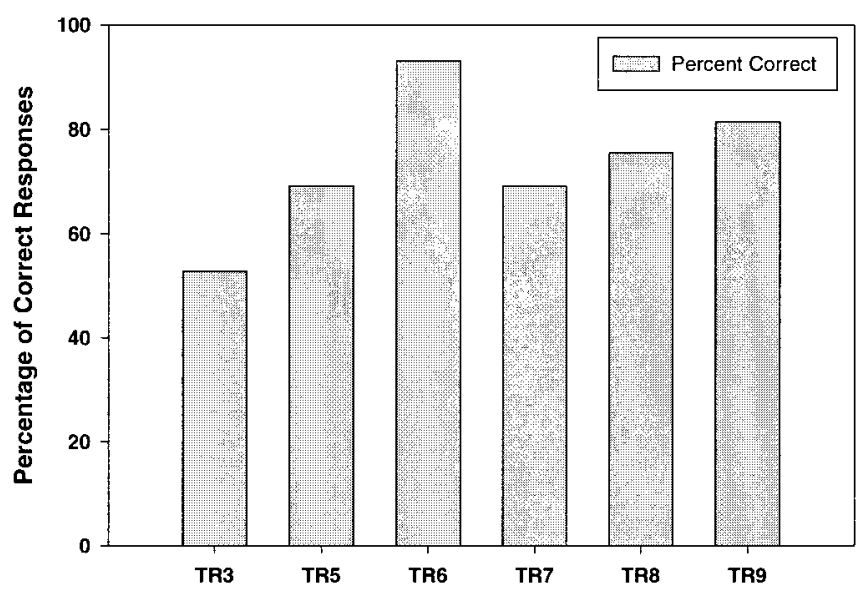

FIG. 4. Histogram showing performance rates for the six experimental conditions. Performance during each experiment was recorded and subsequently analyzed for correctness of response. The percentages of correct responses (number of correct responses as a fraction of the total number of presentations for each condition, summed across the volunteers) are represented in the histogram. There is a notable improvement from $\mathrm{TR}=3 \mathrm{~s}$ to $\mathrm{TR}=6 \mathrm{~s}$, a decrease in performance for the TR $=7 \mathrm{~s}$ and then a gentle increase for $\mathrm{TR}=$ $8 \mathrm{~s}$ and $\mathrm{TR}=9 \mathrm{~s}$.

much of the early work (see, for example, Binder et al., 1994) on fMRI of human auditory cortex was performed with a TR of $3 \mathrm{~s}$. F urthermore, in studies of the motor cortex, among others, a TR of $3 \mathrm{~s}$ is sufficient to

\section{Reaction Times}

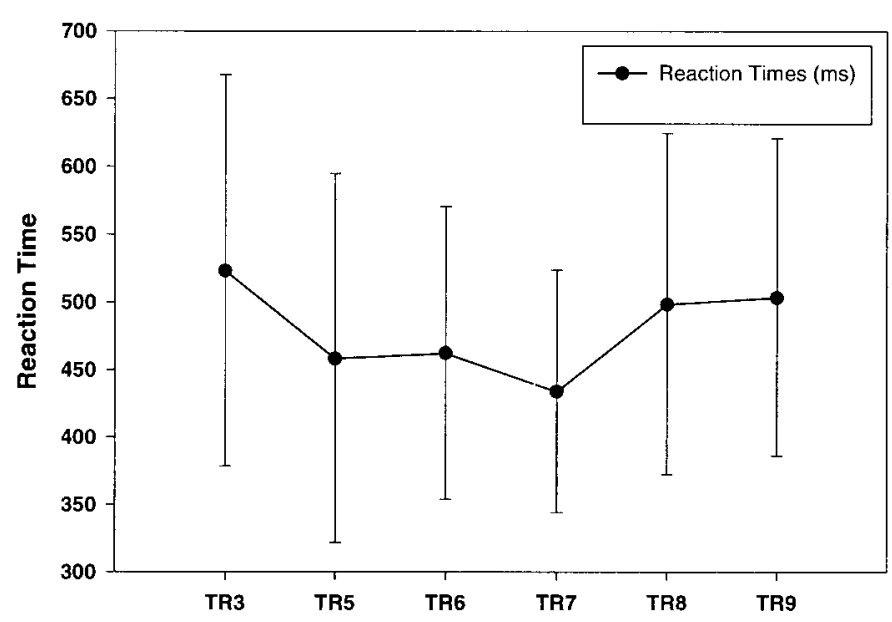

FIG. 5. Histogram showing the averaged reaction times for the six experimental conditions. Although no clear trend is discernible from these data, there is an apparent small drift downward from 3 to $7 \mathrm{~s}$ and then a gentle increase to the TR $=9 \mathrm{~s}$ condition. The standard deviation, as indicated by the error bars, is, however, large and significant changes in reaction time, if any, are small. 
produce a robust BOLD response. The influence of scanner-generated acoustic noise has been investigated recently and shown to have a significant deleterious effect on the BOLD response in auditory experiments (Bandettini et al., 1998; Shah et al., 1999; UImer et al., 1998). The probable cause of the low performance in the TR $=3$ s condition is almost certainly the excessive acoustic noise generated by the scanner which has a masking effect and this is reflected in poor performance and slightly longer reaction times.

The improvement in the t score as TR is increased goes hand in hand with improved performance. Most probably the improved performance results from the fact that during these conditions the duration of the acoustic noise, as a fraction of the total experiment time, is less and this facilitates better task execution. It is noted, however, that the number of target syllables presented is also greater (a consequence of the longer experiment time combined with a constant pre sentation rate) and based on the experimental results presented here, it is not possible to rule that out as an influencing factor.

The levelling off of the t scores for the TR $=8$ and $9 \mathrm{~s}$ conditions coupled with an increasing performance figure seems to suggest that the task became too easy and that the presentation of an increased number of target syllables (216) in a longer experiment may have led to loss of attention. Indeed, during postmeasurement interviews, vol unteers reported some difficulty in concentration during the TR $=9 \mathrm{~s}$ condition. Since it is known that attentional factors play a large role in determining the levels of BOLD activation seen in auditory stimulation experiments (J äncke et al., 1999b), it is possible that activation in the TR $=9 \mathrm{~s}$ condition is lower than it would be if attention had being strongly directed to the task.

The results of this study suggest that the interplay between task-difficulty, fractional duration of acoustic masking noise from the scanner, and attention is important in determining the BOLD response of the auditory cortex. F urther complicating factors, which were deliberately not included in this study, include presentation of the auditory stimuli only during periods free from the acoustic scanner noise. The move to whole brain imaging also presents new dilemmas since the increase in the number of acquired slices (the simplest way to increase coverage and maintain resolution in the third dimension) results in more acoustic masking noise and more intravolume contamination since the whole-brain acquisition time exceeds the BOLD-signal onset time.

A number of recent studies have investigated the use of a variety of different acquisition schemes, either to circumvent the influence of scanner noise or to try and quantify the influence of that acoustic noise. Inevitably, those strategies have led to the use of a variety of repeat times. One study in particular directly investi- gated the effect of sequence repeat time on pure tone thresholds, but only explored rather short TRs (1, 2, and $3 \mathrm{~s}$ ) and found that the change in the threshold to be greatest for the TR $=1 \mathrm{~s}$ sequence. The results of that study are not directly relevant here for two reasons: first, we have chosen a binaural listening task, since this is the one that has been most frequently employed by our group, and second, we have chosen to investigate longer TRs, ranging from 3 to $9 \mathrm{~s}$, because whole-brain scanning is of interest to us and the fMRI community at large. The shortest TR that one can consider for whole-brain scanning at 1.5T with echoplanar imaging is approximately $3 \mathrm{~s}$.

Although the issue of distributed scanning as compared with cluster volume acquisition has been investigated by Edmister et al. (1999) and Talavage et al. (1999), it is not of direct relevance here since in the sense that we have only employed "clustered volume acquisition," by default, since we assume that if the number of slices remains fixed, the duration and character of the gradient noise is also constant. Thus, by changing the repeat time of the sequence, we are in actual fact changing the length of the no-noise period during which stimuli may be presented in the absence of masking effects. It is this issue which we have addressed in order to optimise a frequently-used paradigm. The discussion about the influence of sequence TR cannot, therefore, be seen in isolation from that of acoustic noise. Although we have not directly addressed the issue of separation of intravolume and intervolume noise interference, we have gone some way towards the "sparse" sampling scheme proposed by Hall et al. (1999).

\section{CONCLUSIONS}

Using a phonetic discrimination task performance, the current study demonstrates that the influence of sequence repeat time is considerable. For short TR it is likely that the acoustic noise from the scanner is a significant mask and hinders accurate performance of the task. At the other extreme, a TR of $9 \mathrm{~s}$ is also suboptimal, probably due to attentional effects and lack of concentration and not least because of the longer overall measurement times. The presentation of the total number of target syllables increased as the TR was systematically increased, but contrary to the fact that task performance was reportedly easier, for the longest TR investigated here, $9 \mathrm{~s}$, there was no significant improvement in performance rate and a $t$ score plateau was reached under these conditions. The results of this study point to a complicated interplay between psychophysical factors as well as physical parameters; attention, acoustic noise, total duration of the experiment, consideration of the volume of acquisition, and overall difficulty of the task have to be assessed and balanced. For the paradigm used here, 
the results suggest a TR of around $6 \mathrm{~s}$ for an acquisition (clustered volume), which is limited to 16 slices. Performance data show a high success rate and seemingly the task is difficult enough to hold the attention of subjects and evoke strong and robust BOLD responses from the primary and secondary auditory cortices. Based on the results of these experiments, it is not possibleto rule out an additional influence from the presentation of more target syllables in the conditions with longer TRs.

\section{REFERENCES}

Bandettini, P. A., J esmanowicz, A., Van Kylen, J ., Birn, R. M., and Hyde, J. S. 1998. Functional MRI of brain activation induced by scanner acoustic noise. Magn. Reson. Med. 39:410- 416.

Belin, P., Zatorre, R. J ., Hoge, R., Evans, A. C., and Pike, B. 1999. Event-related fMRI of the auditory cortex. Neurol mage 10:417429.

Binder, J . R. 1997. Neuroanatomy of language processing studied with functional MRI. Clin. Neurosci. 4:87-94.

Binder, J . R., Frost, J . A., Hammeke, T. A., Rao, S. M., and Cox, R. W. 1996a. Function of the left planum temporale in auditory and linguistic processing. Brain 119:1239-1247.

Binder, J . R., Rao, S. M., Hammeke, T. A., Frost, J . A., Bandettini, P. A., and Hyde, J. S. 1994. Effects of stimulus rate on signal response during functional magnetic resonance imaging of auditory cortex. Cogn. Brain Res. 2:31-38.

Binder, J. R., Rao, S. M., Hammeke, T. A., Frost, J . A., Bandettini, P. A., J esmanowicz, A., and Hyde, J . S. 1995. Lateralized human brain language systems demonstrated by task subtraction functional magnetic resonance imaging. Arch. Neurol. 52:593-601.

Binder, J . R., Rao, S. M., Hammeke, T. A., Y etkin, F. Z., J esmanowicz, A., Bandettini, P. A., Wong, E. C., Estkowski, L. D., Goldstein, M. D., Haughton, V. M., et al. 1994. Functional magnetic resonance imaging of human auditory cortex [see comments]. Ann. Neurol. 35:662-672.

Binder, J . R., Swanson, S. J ., Hamecke, T. A., Morris, G. L., Mueller, W. M., Fischer, M., Benbadis, S., Frost, J. A., Rao, S. M., and Haughton, V. M. 1996b. Determination of language dominance using functional MRI: A comparison with the Wada test. Neurology 46:978-984.

Binder, J . R., Frost, J . A., Hammeke, T. A., Cox, R. W., Rao, S. M., and Prieto, T. 1997. Human brain language areas identified by functional magnetic resonance imaging. J . Neurosci. 17(1):353362.

Bregman, A. 1990. Auditory Scene Analysis. MIT Press, Cambridge, MA.

Buckner, R. L., Bandettini, P. A., O'Craven, K. M., Savoy, R. L., Petersen, S. E., Raichle, M. E., and Rosen, B. R. 1996. Detection of cortical activation during averaged single trials of a cognitive task using functional magnetic resonance imaging. Proc. Natl. Acad. Sci. USA 93:14878-14883.

Buckner, R. L., Goodman, J ., Burock, M., Rotte, M., Koutstaal, W., Schacter, D., Rosen, B., and Dale, A. M. 1998. Functional-anatomic correlates of object priming in humans revealed by rapid presentation event-related fMRI. Neuron 20:285-296.

Cho, Z. H., Chung, S. C., Lim, D. W., and Wong, E. K. 1998a. Effects of the acoustic noise of the gradient systems on fMRI: A study on auditory, motor, and visual cortices. Magn. Reson. Med. 39:331335.

Cho, Z. H., Park, S. H., Kim, J . H., Chung, S. C., Chung, S. T., Chung, J. Y., Moon, C. W., Yi, J. H., Sin, C. H., and Wong, E. K. 1997.
Analysis of acoustic noise in MRI. Magn. Reson. Imag. 15:815822.

Counter, S. A., Olofsson, A., Grahn, H. F., and Borg, E. 1997. MRI acoustic noise: Sound pressure and frequency analysis. J . Magn. Reson. Imag. 7:606-611.

Eden, G. F., J oseph, J . E., Brown, H. E., Brown, C. P., and Zeffiro, T. A. 1999. Utilizing hemodynamic delay and dispersion to detect fMRI signal change without auditory interference: The behavior of interleaved gradients technique. Magn. Reson. Med. 41:13-20.

Edmister, W. B., Talavage, T. M., Ledden, P. J ., and Weisskoff, R. M. 1999. I mproved auditory cortex imaging using clustered volume acquisition. Hum. Brain Mapp. 7:89-97.

Friston, K. J . 1995a. Commentary and opinion: II. Statistical parametric mapping: ontology and current issues. J . Cereb. Blood Flow Metab. 15:361-370.

Friston, K. J ., Ashburner, J ., Poline, J . B., Frith, C. D., Heather, J . D., and Frackowiack, R. S. J . 1997. in press. Spatial realigment and normalization of images. Hum. Brain Mapp.

Friston, K. J ., Holmes, A. P., Poline, J . B., Grasby, P. J ., Williman, S. C. R., Frackowiack, R. S. J ., and Turner, R. 1995b. Analysis of fMRI time-series revisited. Neuroimage 2:45-53.

Friston, K. J ., J ezzard, P., and Turner, R. 1994. Analysis of Functional MRI Time-Series. Hum. Brain Mapp. 1:153-171.

Friston, K.J ., Tononi, G., Reeke, G. N., J r., Sporns, O., and E delman, G. M. 1994b. Value-dependent selection in the brain: simulation in a synthetic neural model. Neuroscience 59:229-243.

Friston, K. J ., Worsley, K. J ., Frackowiak, R. S. J ., Mazziotta, J . C., and Evans, A. C. 1994c. Assessing the Significance of Focal Activations Using Their Spatial Extent. Hum. Brain Mapp. 1:210220.

Hall, D. A., Haggard, M. P., Akeroyd, M. A., Palmer, A. R., Summerfield, A. Q., Elliot, M. R., Gurney, E. M., and Bowtell, R. W. 1999. "Sparse" temporal sampling in auditory fMRI. Hum. Brain Mapp. 7:213-223.

J äncke, L., Schlaug, G., Huang, Y., and Steinmetz, H. 1994. Asymmetry of the planum parietale. NeuroReport 5:1161-1163.

J äncke, L., Shah, N. J ., Posse, S., Grosse-Ruyken, M.-L., and MüllerGärtner, H.-W. 1998. Intensity coding of auditory stimuli: An fMRI study. Neuropsycologia 36:875-883.

J äncke, L., Buchanan, T., Lutz, L., Specht, K., Mirzazade, S., and Shah, N. J . 1999. The time course of the BOLD response in human auditory cortex to acoustic stimuli of different duration. Cogn. Brain Res. 8:117-124.

J äncke, L., Mirzazade, S., and Shah, N. J . 1999b. Attention Modulates Activity in the Primary and the Secondary Auditory Cortex: A Functional Magnetic Resonance Imaging Study in Human Subjects. Neurosci. Lett. 266(2):125-128.

J äncke, L., Posse, S., Shah, N. J ., Nösselt, T., Schmitz, N., and Müller-Gärtner, H.-W. 1997. Attentional factors modify the BOLD-response in the human auditory cortex to auditory stimuli. Neuroimage 5:S191. [Abstract]

Millen, S. J., Haughton, V. M., and Yetkin, Z. 1995. Functional magnetic resonance imaging of the central auditory pathway following speech and pure-tone stimuli. Laryngoscope 105:13051310.

Pietrzyk, U., Herholz, K., and Heiss, W.-D. 1990. Three-dimensional alignment of functional and morphological tomograms. J . Comp. Assis. Tomog. 14:51-59.

Pietrzyk, U., Herholz, K., Fink, G., J acobs, A., Mielke, R., Slansky, I., Würker, M., and Heiss, W.-D. 1994. An interactive technique for three-dimensional image registration: Validation for PET, SPECT, MRI and CT brain studies. J. Nucl. Med. 35:2011-2018. 
Poline, J . B., Worsley, K. J ., Holmes, A. P., Frackowiak, R. S., and Friston, K. J . 1995. Estimating smoothness in statistical parametric maps: Variability of $P$ values. J . Comput. Assist. Tomogr. 19: 788-796.

Ravicz, M. E., and Melcher, J. R. 1998. Imager noise and noise attenuation during fMRI. Neurol mage 7:S556.

Shah, N. J ., J äncke, L., Grosse-Ruyken, M.-L., Steinhoff, S., and Müller-Gärtner, H.-W. 1998. The Effect of Sequence Repeat Time on Auditory Cortex Stimulation During Phonetic Discrimination. Neurol mage 7(4):381.

Shah, N. J ., J äncke, L., Grosse-Ruyken, M.-L., and Müller-Gärtner, H.-W. 1999. The influence of acoustic masking noise in fMRI of the auditory cortex during phonetic discrimination. J. Magn. Reson. Imaging 9:19-25.

Talairach, J ., and Tournoux, P. 1988. Co-Planar Stereotaxic Atlas of the Human Brain. 3-Dimensional Proportional System: An Approach to Cerebral Imaging. Thieme Medical Publishers, New York.

Talavage, T. M., Edmister, W. B., Ledden, P. J., and Weiskoff, R. M. 1999. Quantitative assessment of auditory cortex responses induced by imager acoustic noise. Hum. Brain Mapp. 7:89-97.
Ulmer, J . L., Biswal, B. B., Yetkin, F. Z., Mark, L. P., Mathews, V. P., Prost, R. W., Estkowski, L. D., McAuliffe, T. L., Haughton, V. M., and Daniels, D. L. 1998. Cortical activation response to acoustic echo-planar scanner noise. J. Comp. Assis. Tomog. 22:111-119.

Van de Moortele, P. F., Le Clec'h, G., Dehaene, S., and Le Bihan, D. 1998. Improving auditory comprehension in $\mathrm{fMRI}$ : Insertion of silent intervals in multi-slice EPI. Neurol mage 7:S554.

Wessinger, C. M., Buonocore, M. H., Kussmaul, C. L., and Mangun, G. R. 1997. Tonotopy in human auditory cortex examined with functional magnetic resonance imaging. Hum. Brain Mapp. 5:18-25.

Woodruff, P. W. R., Benson, R. R., Bandettini, P. A., Kwing, K. K., Howard, R. J ., Talavage, T., Belliveau, J ., and Rosen, B. R. 1996. Modulation of auditory and visual cortex by selective attention is modality-dependent. Neuroreport 7:1909-1913.

Zatorre, R. J ., J ones-Gotman, M., Evans, A. C., and Meyer, E. 1992. Functional localization and lateralization of human olfactory cortex. Nature 360(6402):339-340.

Zatorre, R. J . Functional neuroimaging in the study of the human auditory cortex [Comment]. Am. J . Neuroradiol. 18(4):621- 623. 